\title{
Erratum
}

\section{Revisiting the Geometry of a Ternary Diagram With the Half-Taxi Metric}

In Figure 2 and within the appendix of my paper concerning the half-taxi metric (Miller, 2002), I expressed the identities for the taxicab cosine function in degrees. They should, instead, have been given in taxicab radians, as in the following:

$$
\cos _{\text {taxi }}\left(\theta_{T}\right)= \begin{cases}1-\frac{\theta_{T}}{2} & \text { if } 0 \leq \theta_{T}<4 \\ -3+\frac{\theta_{T}}{2} & \text { if } 4 \leq \theta_{T}<8\end{cases}
$$

The taxicab radians are defined in terms of the arc length of the diamond-shaped taxicab unit circle, which is shown in Figure 2 of the paper. The essential problem with my presentation is that these identities are incorrect for the Euclidean radians and, unlike degrees and Euclidean radians, there is no simple relationship between degrees and taxicab radians. As noted by Thompson and Dray (2000), taxicab geometry is distinquished from Euclidean geometry by the fact that angles are not rotation invariant. This can be demonstrated by the fact that, in Euclidean geometry, equal central angles sweep out equal lengths of the unit circle. However, equal central angles do not necessarily sweep out equal lengths of the diamond-shaped taxicab unit circle. For example, if we look at the $30^{\circ}$ angle between the position vectors $\mathbf{v}=\left[\begin{array}{ll}1 & 0\end{array}\right]^{\prime}$ and $\mathbf{w}=\left[\begin{array}{ll}\sqrt{3} & 1\end{array}\right]^{\prime}$ in the Cartesian plane and then rigidly rotate the two vectors together through another $30^{\circ}$ angle, the Euclidean radian measure of $\pi / 6$ is unchanged, but the taxicab radian measure changes from approximately 0.732 to 0.536 .

If there is no conversion to degrees, then a derivation, similar to the one supplied within Figure 2, can still be given for the taxicab cosine function in the first quadrant. I apologize for any confusion which these reported identities may have caused. 


\section{REFERENCES}

Miller, W. E., 2002, Revisitng the geometry of a ternary diagram with the half-taxi metric: Math. Geol., v. 34 , no. 3, p. $275-290$.

Thompson, K., and Dray, T., 2000, Taxicab angles and trigonometry: Pi Mu Epsilon J., v. 11, p. 87-96.

W. E. Miller

E-mail:wemo@cdc.gov 